\title{
Lhermitte-Duclos Disease: Incidental Finding in Traumatic Cerebral Hemorrhage
}

\author{
Hemant Kumar Beniwal ${ }^{1}$ Thatikonda Satish ${ }^{1}$ Gollapudi Prakash Rao ${ }^{1}$ Musali Siddartha Reddy ${ }^{1}$ \\ Srikrishnaaditya Manne ${ }^{1}$ \\ ${ }^{1}$ Department of Neurosurgery, Gandhi Medical College and \\ Hospital, Secunderabad, Telangana, India

\begin{abstract}
Address for correspondence Hemant Kumar Beniwal, MBBS, MS, MCh, Department of Neurosurgery, 4th Floor, Gandhi Medical College and Hospital, Padmarao Nagar, Secunderabad 500025, Telangana, India (e-mail: sujanheamnat@gmail.com).
\end{abstract}

Indian J Neurotrauma 2022;19:44-46.

\author{
Abstract \\ Keywords \\ - Lhermitte-Duclos \\ disease \\ - dysplastic cerebellar \\ gangliocytoma \\ - Cowden disease
}

\begin{abstract}
Lhermitte-Duclos disease, also known as dysplastic cerebellar gangliocytoma, is a rare hamartomatous tumor localized in cerebellum. An association with Cowden syndrome is observed in $50 \%$ of cases who present with symptoms of increased intracranial pressure and cerebellar ataxia. These patients have specific magnetic resonance imaging and histopathological findings. Surgical resection is the treatment of choice. Here, we report a case of a young female with traumatic frontal hemorrhage associated with Lhermitte-Duclos disease.
\end{abstract}

\section{Introduction}

Dysplastic cerebellar gangliocytoma, also known as Lhermitte-Duclos disease, is a rare tumor of the cerebellum. These are hamartomatous tumor, may not be neoplastic, classified as World Health Organization (WHO) grade 1 tumor in the current WHO classification of central nervous system (CNS) tumors. ${ }^{1}$ Presentation is typically seen in young adults, in various manners from asymptomatic in smaller lesions to cerebellar signs along with symptoms of raised intracranial pressure, obstructive hydrocephalous in larger lesions. ${ }^{2}$ An association with Cowden syndrome is observed in 50\% of cases. Here, we report a case of traumatic frontal hemorrhage, associated with Lhermitte-Duclos disease.

\section{Case Report}

A 23-year-old female brought to casualty by her relatives with a history of falling while getting down from a bus and sustained a head injury. The patient was referred from a local hospital and on examination the patient was conscious, following commands but intubated with an endotracheal tube with ventilatory support. Patient was tachycardiac with normal blood pressure. Patient was observed in the intensive care unit and extubated after 1 hour.

Thorough reevaluation was done according to trauma guidelines and no other injury was found except the head injury. On a computed tomography (CT) scan, hyperdensity in left frontal region suggestive of acute hematoma is found along with heterogenous hypodensity at right cerebellar region (-Fig. 2). Patient was managed conservatively for the left frontal traumatic hematoma and further evaluated. In background history, patient complained of headache, difficulty in walking, and blurred vision for the last 6 months. On ophthalmoscopy, mild papilloedema was noted suggestive of raised intracranial pressure.

On examination, cerebellar ataxia was found with normal cranial nerve examinations. On the basis of radiological finding of magnetic resonance imaging (MRI) of brain, preoperative diagnosis was made as dysplastic cerebellar gangliocytoma (-Fig. 1). Patient was operated through right suboccipital craniotomy and subtotal excision of tumor was done.

Postoperatively, the patient relieved from her symptoms of blurring vision and headache and cerebellar ataxia was published online July 7, 2021
DOI https://doi.org/

$10.1055 / \mathrm{s}-0041-1729136$

ISSN 0973-0508 (c) 2021. Neurotrauma Society of India.

This is an open access article published by Thieme under the terms of the Creative Commons Attribution-NonDerivative-NonCommercial-License, permitting copying and reproduction so long as the original work is given appropriate credit. Contents may not be used for commercial purposes, or adapted, remixed, transformed or built upon. (https://creativecommons.org/licenses/by-nc-nd/4.0/).

Thieme Medical and Scientific Publishers Pvt. Ltd. A-12, 2nd Floor, Sector 2, Noida-201301 UP, India 


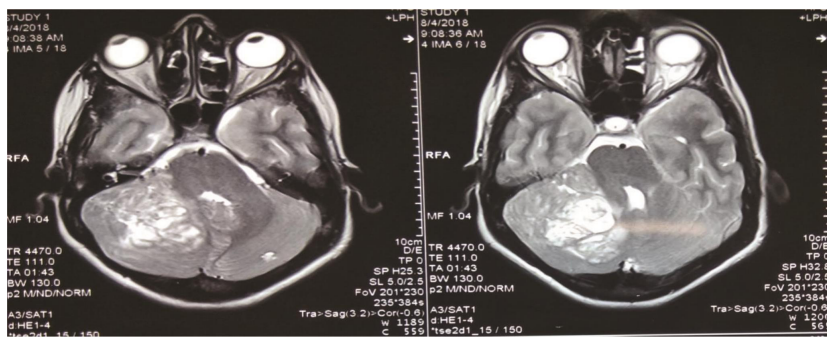

Fig. 1 Hyperintense and tigroid appearance on T2 magnetic resonance imaging.
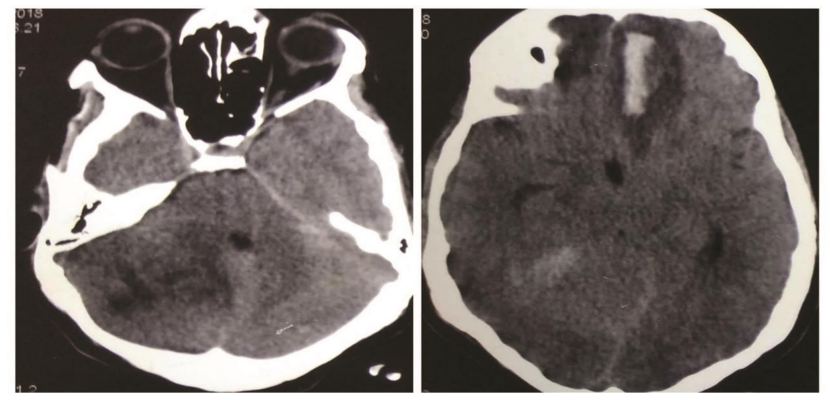

Fig. 2 Noncontrast computed tomography image: Hyperdensity in left frontal region s/o intracranial hemorrhage heterogenous hypodensity in right cerebellar region.
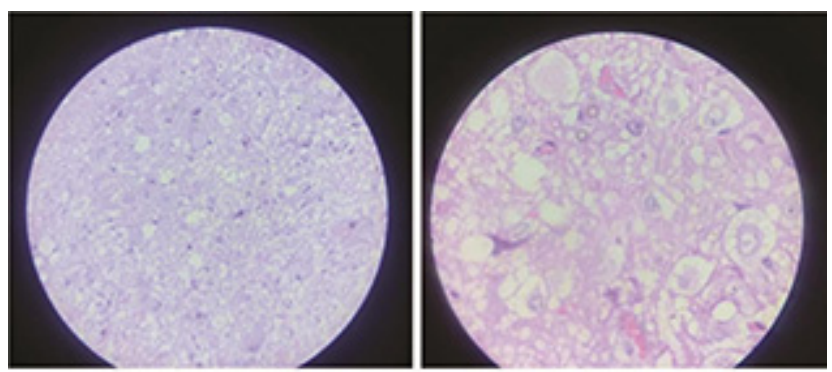

Fig. 3 Histology of pathological cerebellar tissue s/o LhermitteDuclos disease.

improved. On histopathological examination, moderately cellular tissue with groups of ganglion cells with abundant cytoplasm and vesicular nuclei without any mitotic activity suggestive of gangliocytoma was found that supported the preoperative diagnosis (-Fig. 3). On immunohistochemistry synaptophysin and neurofilament protein positivity was found.

On follow-up, after 3 months of surgery, patient was not having any symptoms and cerebellar ataxia also subsided. Dysplastic cerebellar gangliocytoma is a rare lesion which is not reported as an incidental finding in trauma patients in previous studies.

\section{Discussion}

Dysplastic gangliocytoma of cerebellum, also known as Lhermitte-Duclos disease, is a distinctive clinicopathologic entity that is characterized by a cerebellar location, gross enlargement of folia on MRI, and a disorganized cerebellar cortical histology in which large ganglion cells predominate. A layer of myelinated axons in the outermost part of the molecular layer just beneath the pia is also a distinctive feature. An association with Cowden syndrome is observed in $50 \%$ of patients.

Dysplastic cerebellar gangliocytoma is a rare hamartomatous tumor of cerebellar cortex. The pathogenesis of these tumors is poorly understood. These tumors are classified as WHO grade 1 tumor in recent WHO classification of CNS tumors, ${ }^{1}$ although these tumors are not neoplastic. The typical presentation of this disease is found in young adults, but the age of presentation may range from birth to old age. No sex predilection is seen in this disease. ${ }^{3}$

Presentation of Lhermitte-Duclos disease varies from asymptomatic in smaller lesions to cerebellar symptoms like gait disturbances, ataxia, dysmetria, cranial nerve dysfunctions, and increased intracranial pressure symptoms like headache, vomiting, and blurred vision due to obstructed hydrocephalous in the large lesion. An association with Cowden syndrome is observed in $50 \%$ cases that is a rare autosomal dominant disorder, which results from a germline mutation in the PTEN gene on chromosome 10. CNS symptoms associated with Cowden disease may include macrocephaly, heterotopias, seizures, vascular malformations, and mental retardation.

Variety of systemic cancer, including thyroid, breast, and uterine cancers, along with various mucocutaneous lesions is also found with Cowden disease. ${ }^{4}$ The cause of this disease is a controversial issue: origin of disease may be a hamartomatous, neoplastic, or congenital malformation. The close association of dysplastic cerebellar gangliocytoma with multiple hamartoma-neoplasia complex (Cowden syndrome) favors a hamartomatous origin. ${ }^{5}$ These results from dysplastic replacement of the cerebellar Purkinje and granular cells with abnormal ganglion neurons.

In the diagnosis of Lhermitte-Duclos disease, radiological imaging has an important role. CT images show hypoattenuated or isoattenuated cerebellar mass adjacent to normal cerebellum without gross differentiation. CT scan has limited value in diagnosis of this disease. MRI is the modality of choice for the diagnosis of dysplastic gangliocytoma of cerebellum. MRI classically reveals nonenhancing enlargement of cerebellar folia that leads to a striated or tigroid appearance on both T1- and T2-weighted images. Striated bands are iso and hypointense on T1-weighed images and hyper- and isointense on T2-weighted images relative to gray matter. ${ }^{7.8}$

On the basis of the progressive nature of lesion, asymptomatic patients should be followed with serial imaging. In symptomatic patients, surgical resection to relieve mass effect and hydrocephalous is the only effective treatment, ${ }^{8}$ although complete resection is often not possible given the poorly defined margins. Radiotherapy has not been consistently effective for recurrence, ${ }^{8,9}$ which can occur. Although surgery may address obstructive hydrocephalous, cerebellar symptoms often persist. 


\section{Conclusion}

Lhermitte-Duclos disease is a rare hamartomatous tumor localized in cerebellum. An association with Cowden syndrome is observed in $50 \%$ of cases who present with symptoms of increased intracranial pressure and cerebellar ataxia. In our case, patient presented with traumatic cerebral hematoma with background cerebellar dysplastic gangliocytoma that may or may not cause the traumatic incident.

\section{Funding}

None.

\section{Conflict of Interest}

None declared.

\section{References}

1 Louis DN, Ohgaki H, Wiestler OD, et al. The 2007 WHO classification of tumours of the central nervous system. Acta Neuropathol 2007;114(2):97-109
2 Pandey S, Sarma N. Lhermitte-Duclos disease: a rare cause of cerebellar ataxia. Asian J Neurosurg 2017;12(4):705-706

3 Nowak DA, Trost HA, Porr A, Stölzle A, Lumenta CB. Lhermitte-Duclos disease (Dysplastic gangliocytoma of the cerebellum). Clin Neurol Neurosurg 2001;103(2):105-110

4 Robinson S, Cohen AR. Cowden disease and Lhermitte-Duclos disease: characterization of a new phakomatosis. Neurosurgery 2000;46(2):371-383

5 Nowak DA, Trost HA. Lhermitte-Duclos disease (dysplastic cerebellar gangliocytoma): a malformation, hamartoma or neoplasm? Acta Neurol Scand 2002;105(3):137-145

6 Milbouw G, Born JD, Martin D, et al. Clinical and radiological aspects of dysplastic gangliocytoma (Lhermitte-Duclos disease): a report of two cases with review of the literature. Neurosurgery 1988;22(Pt 1):124-128

7 Meltzer CC, Smirniotopoulos JG, Jones RV. The striated cerebellum: an MR imaging sign in Lhermitte-Duclos disease (dysplastic gangliocytoma). Radiology 1995;194(3):699-703

8 Borni M, Kammoun B, Kolsi F, Abdelmouleh S, Boudawara MZ. The Lhermitte-Duclos disease: a rare bilateral cerebellar location of a rare pathology. Pan Afr Med J 2019;33:118

9 Bozbuga M, Gulec I, Suslu HT, Bayindir C. Bilateral Lhermitte-Duclos disease. Neurol India 2010;58(2):309-311 